\title{
Nonconjugated Polyelectrolyte as Efficient Fluorescence Quencher and Their Applications as Biosensors: Polymer-Polymer Interaction
}

\author{
${\text { Vidya } \text { Raj }^{1} \text { and Sreenivasan Kunnetheeri }}^{2}$ \\ ${ }^{1}$ Department of Chemistry, Indian Institute of Space Science and Technology, Department of Space, Valiyamala, \\ Trivandrum 695547, India \\ ${ }^{2}$ Laboratory for Polymer Analysis, Biomedical Technology Wing, Sree Chitra Tirunal Institute for Medical Sciences and Technology, \\ Poojapurra, Trivandrum 695012, India
}

Correspondence should be addressed to Sreenivasan Kunnetheeri; sreeni@sctimst.ac.in

Received 9 October 2013; Accepted 24 November 2013; Published 22 January 2014

Academic Editors: M. E. Lorenzo and I. Zhukov

Copyright (C) 2014 V. Raj and S. Kunnetheeri. This is an open access article distributed under the Creative Commons Attribution License, which permits unrestricted use, distribution, and reproduction in any medium, provided the original work is properly cited.

\begin{abstract}
A simple fluorescence quenching method for the quantitation in serum of an acute phase reactant, C-reactive protein (CRP), which can differentiate between viral and bacterial infections, is described, where material and reagent costs are minimal. The study harnesses a fluorescence quenching between a nonfluorescent polyelectrolyte containing a ligand (O-phosphorylethanolamine, PEA) and fluorophore (fluorescamine Isomer 1) containing polyelectrolyte. The quenching was attributed due to strong polymerpolymer interaction through intermolecular hydrogen bonding. The nonlinear behaviour of Stern-Volmer plot indicates a binding induced quenching, that is, static quenching. However, fluorescence was found to increase in presence of C-reactive protein, due to the specific molecular recognition occurring between CRP and PEA, thereby excluding fluorophore containing chain. A definite correlation was found between concentration of CRP and fluorescence intensity and the method exhibited a linear relationship in the range of $40-360 \mathrm{ng} / \mathrm{mL}$ with a detection limit of $30 \pm 2 \mathrm{ng} / \mathrm{mL}$. The antibody free method was successfully applied for the analysis of CRP in human serum samples and the method showed good correlation with hospital measurements $(y=1.0313 x-0.1423 ; n=$ 32; $R=0.9998, P<0.0001)$. Thus the fluorescence based polyelectrolyte biosensor is a potential system for rapid, and antibody free platform for CRP detection.
\end{abstract}

\section{Introduction}

Polyelectrolytes based fluorescent sensors have been an area of intense research for the past few decades, due to the interest in area such as controlled drug release, surface coating, and chemical and biological sensing [1-5]. Conjugated polyelectrolytes have received particular attention for sensing proteins, due to their water solubility and fluorescence properties [6-9]. Conjugated polyelectrolytes contain a set of structural attributes that make them useful in optical and electronic detection of many chemical and biological targets [10]. Fluorescence methods are being used for a variety of investigations in biochemical, medical, and chemical research. Fluorescence based sensors may rely on changes in emission intensity, life time, and wavelength (excitation and emission).
However, fluorescence quenching, in which decrease of emission intensity and/or life time is measured, is one of the most common techniques in fluorescence based sensors because of its easy measurement and low detection limit.

Herein we report a nonconjugated polyelectrolyte as efficient fluorescent quencher and its application for the quantitation in serum of an acute phase reactant, $\mathrm{C}$-reactive protein (CRP). In this investigation, we present a pioneering analytical platform integrating the properties of polyelectrolyte and fluorescence quenching mechanisms. As a platform model, measurement of CRP a cardiac inflammation marker was performed.

CRP is one of the well studied marker molecules in association with cardiovascular diseases (CD) [11]. CRP has been reported to be elevated up to 1000 folds during 
many infectious states including myocardial infarction [12]. CRP consists of five identical subunits each of which is formed of 206 amino acid units [13]. It has binding sites for phosphocholine and its derivatives. The binding is known to be calcium-dependent. Recent studies are suggestive that CRP along with serum cholesterol is decisive factors in the initiation and progression of CD [14]. A growing body of evidence has suggested that considerable prospect exists in using CRP measurement as a predictor of possible cardiovascular risks.

Several methodologies have been reported for its estimation [15-18] and among the reported methods, immunoassays enjoy wide acceptance due to their specificity and sensitivity $[19,20]$. One of the serious concern of these methodologies are associated with the often noted nonspecific binding and nonstability of the conjugated biomacromolecules (e.g., antibodies) [21]. Recently several measures have been taken to take care of these factors.

Efforts to develop techniques using nonbiological components, interestingly, are scanty. Merritt and Winkelman have reported an electrochemical sensor for CRP based on crown ether [22]. Apart from these report chemical sensors for the detection of CRP have not yet been reported. Here we report a new analytical platform for sensing CRP in human sera based on the fluorescence quenching of a pair of copolymers. The sensor platform described here could be modified for the detection of other clinically interesting molecules by the attachment of appropriate functionalities.

\section{Experimental}

2.1. Materials. Acrylic acid $(\mathrm{Mw}=7206)$ and fluorescamine isomer 1 were purchased from Acros Organics, New Delhi, India. O-phosphorylethanolamine, 1-ethyl-3-(3-dimethylaminopropyl) carbodiimide hydrochloride (EDC), human albumin, azobisisobutyronitrile (AIBN), and human C-reactive protein were obtained from Sigma-Aldrich chemicals Inc., Bangalore, India. These chemicals were used as received. Other analytical/chromatographic grade solvents were purchased from Merck or Sisco Chemicals, Mumbai, India

2.2. Homopolymerisation of Acrylic Acid. Briefly, acrylic acid (5 g) was homopolymerised in $50 \mathrm{~mL}$ benzene using AIBN as initiator at $60^{\circ} \mathrm{C}$ in an atmosphere of nitrogen. After $6 \mathrm{~h}$, the homopolymer obtained (F0) was washed and dialysed against water and lyophilised.

2.3. Coupling of PEA to Polyacrylic Acid. $1 \mathrm{~g}$ of homopolymer was dissolved in water and the $\mathrm{pH}$ of the solution was adjusted to be around 4.5. Carbodiimide chemistry was used to form amide bond between carboxylic group of polyacrylic acid and primary amine group of PEA as depicted in Scheme 1(a) [23-25]. As a first step, EDC reacts with a carboxylic acid group and forms an amine reactive intermediate. In the next step, addition of a primary amine containing molecule in this reaction mixture leads to an amide linkage between amine and the activated carboxylic acid group. The activation is generally done at low $\mathrm{pH}(\mathrm{pH} \sim 4.5)$ because at this $\mathrm{pH}$ acid group gets protonated. Biochemists use this procedure to link biomolecules or to modify biomolecule surfaces. PEA conjugated polymer was dialysed against water, lyophilized, and stored. The polymer was designated as F1.

2.4. Coupling of Fluoreseamine Isomer 1 to Polyacrylic Acid. $\mathrm{g}$ of homopolymer was dissolved in water and the $\mathrm{pH}$ of the solution was adjusted to be around 4.5. Carbodiimide chemistry was used to form amide bond between carboxylic group of polyacrylic acid and primary amine group of fluorophore as depicted in Scheme 1(b). The fluorophore anchored polymer was dialysed against water and lyophilised. The polymer was abbreviated as F2.

2.5. Interaction of CRP with Polyelectrolytes F1 and F2. The analysis of protein interaction with the polyelectrolyte was performed by adding $20 \mathrm{mg}$ of $\mathrm{F} 2$ in $50 \mathrm{~mL}$ of $0.1 \mathrm{M}$ calcium chloride solution for the measurement of fluorescence. Exactly $20 \mathrm{mg}$ of F1 was added to the same solution for the subsequent measurement. Varied amount of CRP were added to the solution $(10 \mathrm{~mL})$ containing mixture of F1 and F2. All the measurements were made in triplicate and the average readings were taken. The excitation wavelength was $496 \mathrm{~nm}$ and the emission was $520 \mathrm{~nm}$. The relative intensities were corrected for dilution.

2.6. Interference Studies with a Mixture of Albumin, Fibrinogen, and $\gamma$ Globulin. A $10^{-2} \mathrm{M}$ stock solution of each protein (albumin, fibrinogen and $\gamma$-globulin) was prepared in phosphate buffer solution of $\mathrm{pH}$ 7. It is well known that concentration of albumin in the blood is $50 \mathrm{mg} / \mathrm{mL}$. The levels of other proteins are relatively lower. Since the volume of serum we used for the measurement was $10 \mu \mathrm{L}$, we added a mixture of proteins $(500 \mu \mathrm{g} / \mathrm{mL})$ in which the concentration of albumin was kept at $480 \mu \mathrm{g} / \mathrm{mL}$ to assess the interference in the measurement of CRP.

2.7. Analysis of CRP in Human Sera. The levels of CRP in human sera were determined by two different methods: the fluorescence quenching method and by the method of nephelometry employing CRP specific antibodies. For the analysis of CRP by fluorescence method, ten microlitres of the serum separated from the blood samples were added into $2 \mathrm{~mL}$ of the copolymer solution. The copolymer solution was prepared by dissolving $10 \mathrm{mg}$ each of polymers (F1 and F2) in $25 \mathrm{~mL}$ of phosphate buffer, $\mathrm{pH} 7$, containing $0.1 \mathrm{M}$ calcium chloride. The solution was shaken well and the fluorescence was measured at $22^{\circ} \mathrm{C}$. All the measurements were made in triplicate and the average value was taken. Then, correlation between two methods was analysed with the Linear Fit of the Origin program.

2.8. Instrumental. The infrared spectrum was measured in the range $400-4000 \mathrm{~cm}^{-1}$ using a Nicolet Inc. (Madison, USA) model impact 5700 FTIR spectrometer with a horizontal ATR accessory containing diamond crystal. The numbers of scans were 50 . 


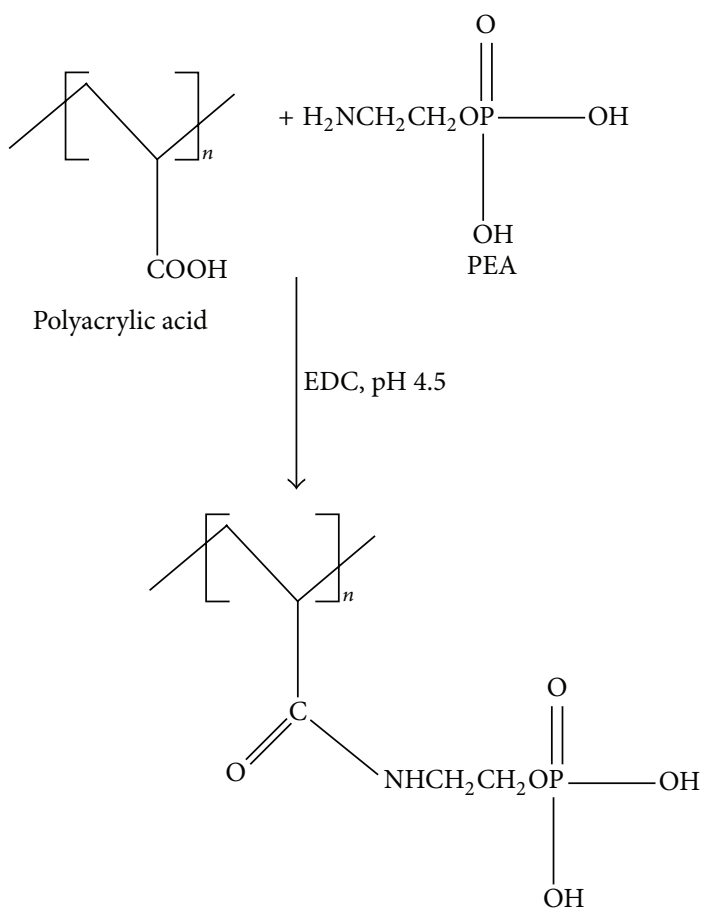

PEA conjugated polyacrylic acid

(a)

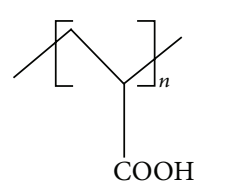

Polyacrylic acid
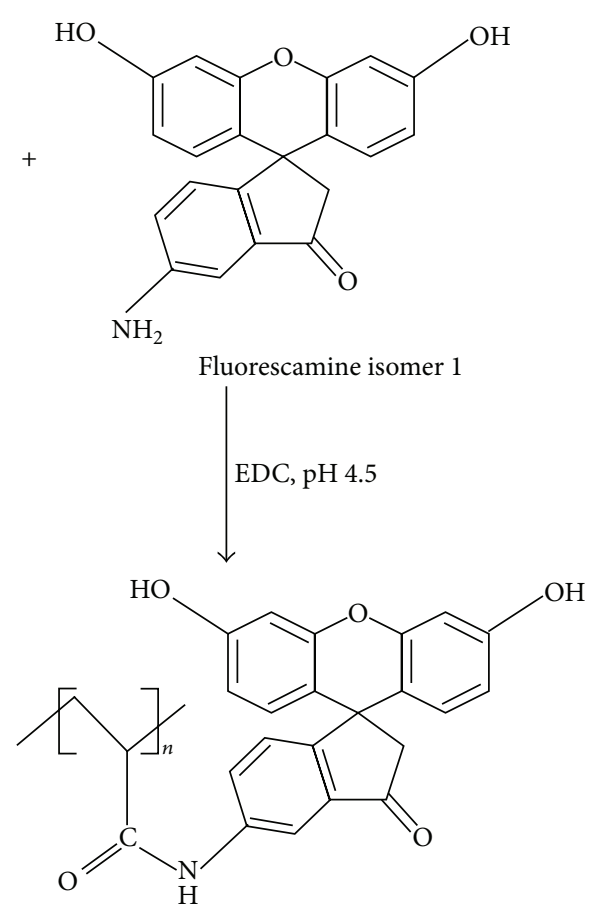

Fluorophore conjugated polyacrylic acid

(b)

SCHEME 1: (a) Schematic representation of formation of PEA conjugated polyacrylic acid by EDC chemistry and (b) schematic representation of formation of fluorophore conjugated polyacrylic acid.

The fluorescence spectrum was recorded using a Cary Eclipse model EL 0507 spectrofluorimeter.

Energy dispersive X-ray analysis was performed using an EDX model 6051 SP (Oxford Instruments, UK) attached to the scanning electron microscope.

MININEPH (Binding site Ltd., Birmingham, UK) was used for determining CRP concentration in serum using the principle of nephelometry. The light source is a diode laser that emits at $670 \mathrm{~nm}$. The focussed light passes through a cuvette containing the reaction mixture, where antibody/antigen complexes cause light to be scattered. This scatter is proportional to the amount of antibody/antigen complexes that have formed and is detected by a photodiode. For each assay, a scatter is taken at the beginning of the antibody/antigen reaction (Blank), followed by a second scatter reading at a fixed time. The analyte concentration is calculated using the difference between these two readings. The measuring range is $3.5-112 \mathrm{mg} / \mathrm{L}(3.5-112 \mu \mathrm{g} / \mathrm{mL})$.

\section{Results and Discussion}

Polyelectrolyte based sensory materials are one among them which has been widely used in the area of protein sensing or more precisely for biomolecular recognition. For the most part, this growth has been driven by the use of conjugated polyelectrolytes that are functionalised with appropriate side chains or ligands. Contrary to these reports we demonstrate the use of nonconjugated polyelectrolyte as fluorescence quencher and its application in the sensing of CRP. Towards this goal we have synthesised two polymeric formulations: (1) polyacrylic acid conjugated with PEA and (2) polyacrylic acid on conjugated with fluoreseamine Isomer 1.

3.1. Characterisation of Polyelectrolytes F1 and F2 (Fourier Transform Infrared Spectroscopy (FTIR)). Figure S1 (see supplementary information in the Supplementary Material available online at http://dx.doi.org/10.1155/2014/841857) shows the infrared spectrum of these polymers. PEA conjugated polymer (spectrum B) showed peak around $1553 \mathrm{~cm}^{-1}$ characteristic of amide bond (-CONH), confirming the coupling PEA onto polyacrylic acid through the activated carboxylic acid groups. The intensity of $-\mathrm{CO}$ and $-\mathrm{OH}$ groups were reduced substantially further reflecting the formation PEA conjugated entities through the carboxylic acid groups. The spectrum also showed a peak around $1000 \mathrm{~cm}^{-1}$ characteristic of $\mathrm{PO}_{4}{ }^{-}$moiety further confirming the result. Similarly the spectrum of fluorescamine conjugated polymer (spectrum C) showed distinctive peak around $1550 \mathrm{~cm}^{-1}$ characteristic of amide bond formation between the $-\mathrm{NH}_{2}$ group of fluorophore and the carboxylic acid group of polyacrylic acid. A strong peak around $1110 \mathrm{~cm}^{-1}$, characteristic of C-O-C of fluorescamine further indicates the formation fluorophore coupled polymer.

3.2. Energy Dispersive X-Ray Analysis (EDAX). EDX trace depicted in Figure S2 (Supplementary Information) shows the peak corresponding to phosphorous indicating the conjugation of phosphate moieties onto polyacrylic acid. Peaks of 


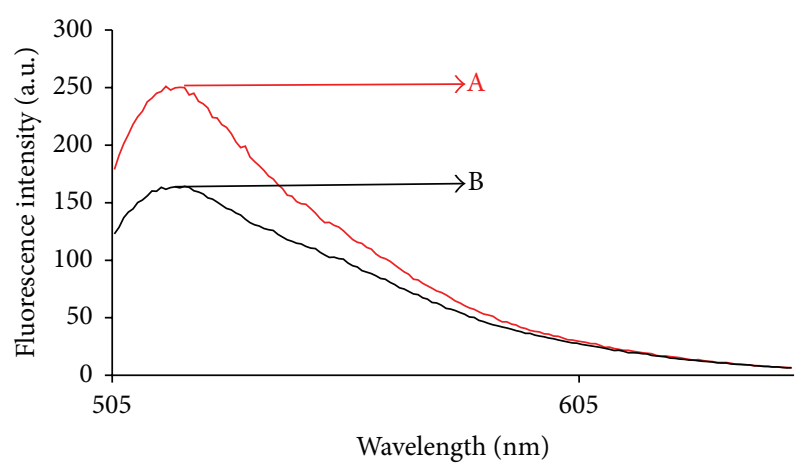

FIGURE 1: Fluorescence spectrum of F2 (trace A), fluorescence spectrum of F1 + F2 (trace B).

$\mathrm{Au}$ and Pd can also be seen in the trace which is arisen from the coated gold layer prior to the observation.

3.3. Fluorescence Quenching Experiments. The emission spectrum of F2 is shown in Figure 1 (trace A). An equal quantity of F1 was added to the solution containing F2 and the reaction mixture was shaken well. The fluorescence measurements indicated a decrease in the fluorescence spectrum of F2 or in other words the fluorescence seems to be quenched (Figure 1, trace B). F1 quenched nearly $40 \%$ of the initial polymer fluorescence. The drastic quenching of polymer fluorescence of F2 by F1 could be traced due to strong polymer-polymer interaction. Kabanov et al. have studied in detail the association of polymeric chains in aqueous solution using fluorescence [26]. In our system of nonconjugated polyelectrolyte the possibility of energy transfer or electron transfer is completely ruled out. The quenching of fluorescence was attributed due to the strong interactions between polymeric chains due to intermolecular hydrogen bonding. Difference in quenching efficiency between different groups can be attributed to sterric, electrostatic, and hydrophobic interactions inherent to the differences in the molecular structure [27]. Here since PEA is a small molecule it is easily associated with the polymer structure.

Because of the charged groups on the backbone, chainchain interactions in water soluble polyelectrolyte are subjected to some interpolymer forces due to hydrogen bonding. The interaction of these chains seems to curtail the fluorescence since the fluorophore is buried to an altogether different environment. When polyelectrolytes are mixed in water, the backbone of the two polymers interacts, which brings the fluorophore and PEA in close proximity to the optically active backbone. Under these circumstances, the fluorescence quenching of $\mathrm{F} 2$ by $\mathrm{F} 1$ is very efficient, or in other words when the two polyelectrolytes (F1 and F2) are mixed in water the system behaves as a "turn-off" sensor (Scheme 2(a)).

The fluorescence quenching efficiencies can be quantified using Stern-Volmer equation which is given by

$$
\frac{F_{0}}{F}=1+K_{\mathrm{sv}}[Q]
$$

where $F_{0}$ and $F$ are fluorescence intensities in the absence and presence of quencher and $[Q]$ is the quencher concentration.

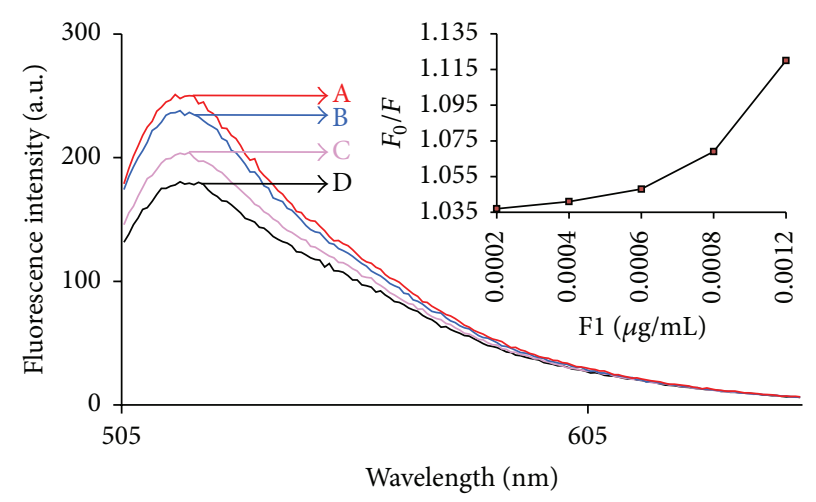

FIGURE 2: Fluorescence spectra in presence of different quencher concentrations. Inset is the Stern-Volmer plot.

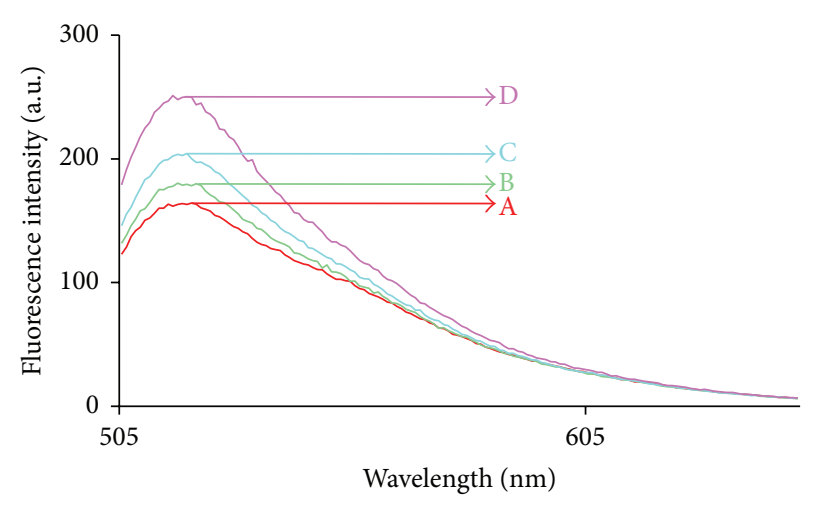

FIGURE 3: Effect of varied concentration of CRP on the fluorescence spectrum of F1 + F2. Fluorescence spectrum of F1 + F2 (trace A), fluorescence spectrum of F1 + F2 + $120 \mathrm{ng} / \mathrm{mL}$ CRP (trace B), fluorescence spectrum of F1 + F2 + $240 \mathrm{ng} / \mathrm{mL}$ CRP (trace C), and fluorescence spectrum of F1 + F2 + $360 \mathrm{ng} / \mathrm{mL}$ CRP.

The Stern-Volmer constant $K_{\mathrm{sv}}$ provides a direct measure of the quenching efficiencies and is determined from the linear portion of the plot $F_{0} / F$ versus [Q]. Concentrationdependent quenching was used to generate a Stern-Volmer plot (Inset, Figure 2). For PEA induced fluorescence quenching, this value was found to be $1.8 \times 10^{2}$. The nonlinear behaviour of the plot indicates a binding induced fluorescence quenching that is, a static quenching.

3.4. Fluorescence Recovery by CRP. When F1 and F2 are allowed to interact with solution, the polyelectrolytes associate to give a fluorescence quenching effect as explained earlier. The addition of CRP, which selectively binds the PEA moieties on F1, was described to form a complex with PEA and thereby excludes the polymeric chain containing fluorophore. The specific molecular recognition between PEA and CRP draws away polyelectrolytic chain containing fluorophore, thereby recovering the emission (Figure 3).

In other words, the average distance between ligand and the fluorophore is larger upon addition of CRP since upon conjugation of PEA with CRP pulls the polymeric chain containing the ligand towards itself, and hence the polymeric chain containing the fluorophore is excluded thus 

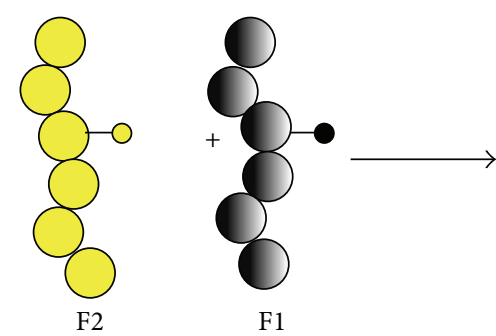

O Flurophore

- PEA

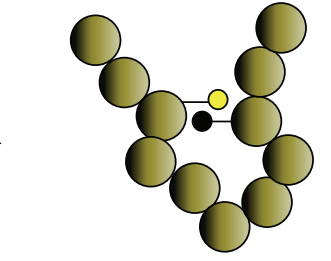

Fluorescence "turn off" Polymer chain-chain interaction
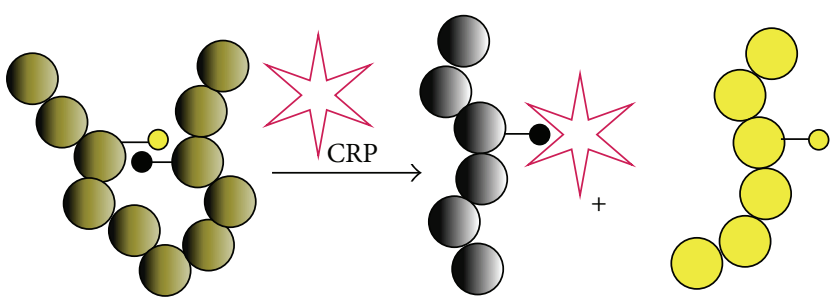

Fluorescence "turn off"

Polymer chain-chain interaction

PEA

Fluorophore

(a)

(b)

SCHEme 2: (a) Schematic representation of "turn-off" sensor and (b) schematic representation of "turn-on" sensor system developed for CRP detection.

functioning as a "turn-on" system. A feasible mechanism for variation in fluorescence intensity is represented in Scheme 2(b). Regaining fluorescence emission upon addition of avidin has been reported by Shi and Wudl, in which the fluorescence emission of poly [lithium2-methoxy-5( $3^{\prime}$-propyloxylsulfonate)-1,4-phenylenevinylene] $\left(\mathrm{Li}^{+}\right.$-MPS$\mathrm{PPV})$ is quenched by a probe molecule [N-(biotinoyl)- $\mathrm{N}^{\prime}$ (acetyl 4,4' pyridylpyridinium iodide)] $\left(\mathrm{BPP}^{+}\right)$. The addition of avidin which selectively binds the biotin substructure encapsulate $\mathrm{BBP}^{+}$and draw it away from $\mathrm{Li}^{+}$-MPS-PPV, thereby recovering the emission [28]. With different concentrations of CRP, the polymer fluorescence continues to grow up to $[\mathrm{CRP}]=360 \mathrm{ng} / \mathrm{mL}$. Further CRP addition (upto $360 \mathrm{ng} / \mathrm{mL}$ ) does not result in additional changes in emission intensity. When all the binding sites on PEA are saturated with CRP, further addition of CRP results in the unperturbed fluorescence emission.

3.5. Effect of Nonspecific Proteins on the Fluorescence Quenching of $F 2$ by $F 1$. In a realistic analysis, a given sample may contain nontargets species that should not perturb the diagnostic signals of the sensory mechanism. Since PEA showed specificity for CRP binding, it was of interest to examine that other blood proteins would perturb the fluorescence. For the purpose of the study we chose three other blood proteins namely, albumin, fibrinogen, and $\gamma$-globulin. The addition of three different proteins to a quenched polymer solution (Figure 4, trace A) displayed unperturbed fluorescence emission as indicated in Figure 4 (trace B). Though we have not attempted to probe why albumin is repelled from the polymer surface, it seems that the presence of PEA moieties is responsible for the nonuptake of albumin. Park et al. have recently shown that binding of albumin onto polymeric nanoparticles could be reduced remarkably by modifying the surface by phosphorylcholine moieties [29]. Whereas addition of CRP to the same solution resulted in specific recovery of the emission as indicated in trace C (Figure 4). These results suggest that none of the blood proteins other than CRP has the capacity to bind to PEA, and thus the recovery seen from the polyelectrolyte/CRP solutions can only be due to the specific interaction between PEA and CRP moieties.

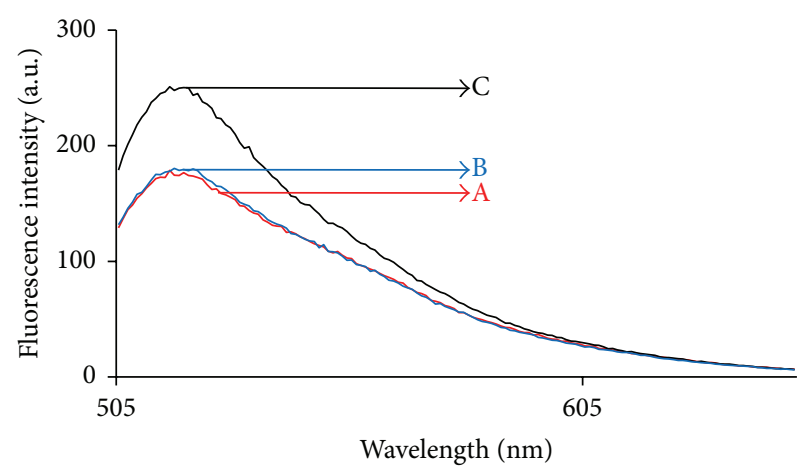

FIGURE 4: Fluorescence Spectrum of F1 + F2 (trace A), F1 + F2 + nonspecific proteins (trace $\mathrm{B}$ ), and $\mathrm{F} 1+\mathrm{F} 2+$ nonspecific proteins + CRP (trace C and D).

3.6. Measurement of CRP in Real Blood Samples. With the initial concept and development of new sensor platform, the next step was demonstrating the system in real blood samples. In order to accomplish this blood samples collected from various patients reported to the cardiology were tested using the method of polyelectrolyte and the values were compared with the values obtained for the same sample by normal CRP diagnostic test using CRP specific antibody. Fluorescence intensity increases only marginally when a negative CRP sample (samples containing CRP less than $3 \mathrm{mg} / \mathrm{L}$ ) was added to the quenched polymer solution (Figure 5 , trace B). However, in presence of positive samples (samples containing CRP higher than $3 \mathrm{mg} / \mathrm{L}$ ), fluorescence intensity increases appreciably as depicted in Figure 5 , traces $C$ and D. The amount of CRP in various serum samples is summarised in Table 1 . The quantity of CRP in these samples estimated using immunoassay is also shown in Table 1. The data suggest that the present method based on polyelectrolyte is indeed comparable with the well-known immunoassay technique.

3.7. Analysis of CRP in Human Sera: Correlation between Methods. The correlation between our method and conventional CRP nephelometry technique employed at our hospital 


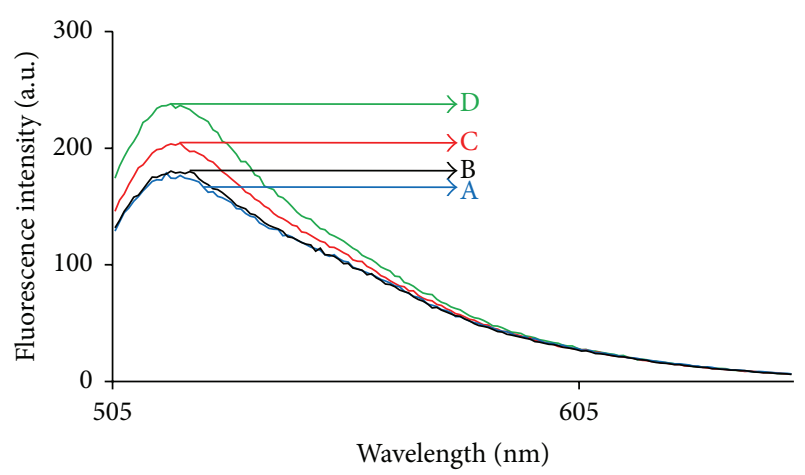

Figure 5: Fluorescence spectrum of F1 + F2 (trace A), F1 + F2 + negative serum samples (trace $B$ ), and $F 1+F 2+$ positive serum samples (trace $\mathrm{C}$ and trace $\mathrm{D}$ ).

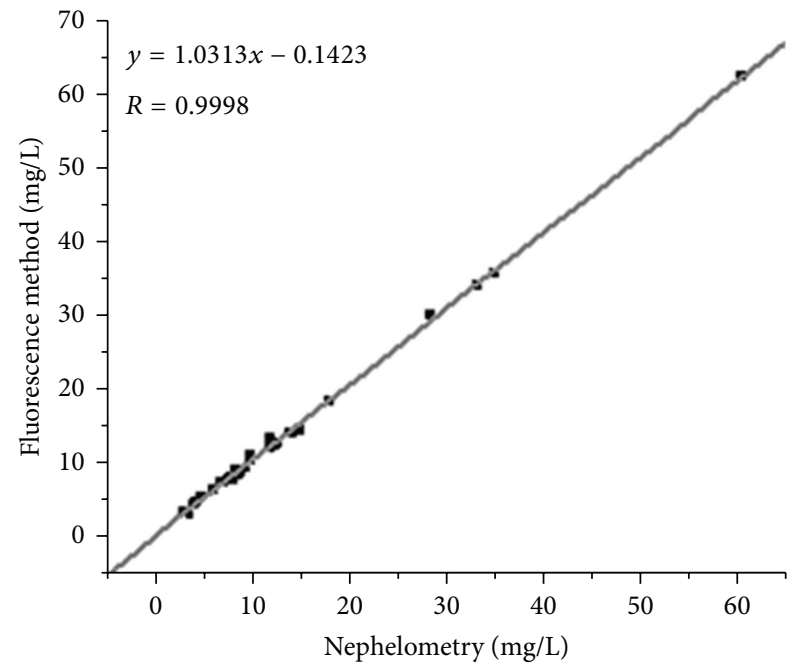

FIGURE 6: Analysis of CRP in human sera. Correlation between the CRP levels determined by nephelometry and by fluorescence was analysed by the Fit Linear of the Origin program $(n=32$; $R=0.9998 ; P<0.0001)$.

laboratory (Department of Biochemistry, Sree Chitra Tirunal Institute for Medical Sciences and Technology) was investigated. Accordingly, 40 blood patient samples (32 positive and 8 negative CRP samples) were determined by conventional nephelometry and by the method of fluorescence as described in Section 2. Then, correlation between the CRP levels determined by the method of nephelometry and those determined by fluorescence was analysed by the Fit Linear of the Origin program. As shown in Figure 6, the CRP levels of human sera determined by nephelometry showed a good correlation with those determined by fluorescence $(n=32 ; R=0.9998$; $P<0.0001)$ indicating the clinical relevance of our results. The correlation is described by the following equation: $y=$ $1.0313 x-0.1423 ; n=32$. The mean difference of the methods according to the Bland and Altman plots is 0.4699 $\pm 6.45 \mathrm{ng} / \mathrm{mL}$ (Figure $7(\mathrm{a})$ ). To get further insight into the affinity of PEA to CRP, binding affinity has been calculated from a dose-response curve constructed from the adsorption data by Langmuir fitting (Figure 7(b)). The binding constant
TABLE 1: Quantitative data of CRP in serum-comparison of the methods.

\begin{tabular}{|c|c|c|}
\hline Sample code & $\begin{array}{c}\text { Amount of CRP } \\
\text { nephelometry }(\mathrm{mg} / \mathrm{L})\end{array}$ & $\begin{array}{c}\text { Amount of CRP } \\
\text { fluorescence }(\mathrm{mg} / \mathrm{L})\end{array}$ \\
\hline 1 & 4.58 & $4.73 \pm 0.15$ \\
\hline 2 & 15.04 & $14.44 \pm 0.09$ \\
\hline 3 & 14.11 & $14.25 \pm 0.50$ \\
\hline 4 & 35.01 & $35.63 \pm 0.10$ \\
\hline 5 & 28.66 & $30.17 \pm 0.21$ \\
\hline 6 & 15.07 & $15.42 \pm 0.23$ \\
\hline 7 & 12.84 & $12.76 \pm 0.45$ \\
\hline 8 & 7.15 & $7.34 \pm 0.12$ \\
\hline 9 & 12.00 & $13.45 \pm 0.14$ \\
\hline 10 & 8.23 & $7.79 \pm 0.05$ \\
\hline 11 & 8.76 & $8.76 \pm 0.55$ \\
\hline 12 & 8.44 & $8.56 \pm 0.09$ \\
\hline 13 & 33.45 & $34.10 \pm 0.11$ \\
\hline 14 & 18.26 & $18.47 \pm 0.41$ \\
\hline 15 & 7.06 & $7.45 \pm 0.21$ \\
\hline 16 & 9.58 & $9.19 \pm 0.10$ \\
\hline 17 & 14.12 & $14.21 \pm 0.90$ \\
\hline 18 & 8.56 & $9.87 \pm 0.15$ \\
\hline 19 & 10.07 & $10.98 \pm 0.22$ \\
\hline 20 & 12.04 & $12.14 \pm 0.55$ \\
\hline 21 & 4.82 & $4.97 \pm 0.12$ \\
\hline 22 & 8.97 & $8.79 \pm 0.15$ \\
\hline 23 & 17.72 & $17.64 \pm 0.24$ \\
\hline 24 & 7.94 & $7.64 \pm 0.11$ \\
\hline 25 & 4.95 & $4.25 \pm 0.15$ \\
\hline 26 & 10.15 & $10.45 \pm 0.20$ \\
\hline 27 & 16.29 & $16.45 \pm 0.11$ \\
\hline 28 & 14.36 & $14.12 \pm 0.16$ \\
\hline 29 & 12.48 & $12.24 \pm 0.15$ \\
\hline 30 & 60.41 & $62.45 \pm 0.24$ \\
\hline 31 & 12.97 & $12.21 \pm 0.14$ \\
\hline 32 & 17.9 & $16.73 \pm 0.09$ \\
\hline 33 & Negative sample & $\begin{array}{l}\text { Marginal change in } \\
\text { fluorescence }\end{array}$ \\
\hline 34 & Negative sample & $\begin{array}{l}\text { Marginal change in } \\
\text { fluorescence }\end{array}$ \\
\hline 35 & Negative sample & $\begin{array}{l}\text { Marginal change in } \\
\text { fluorescence }\end{array}$ \\
\hline 36 & Negative sample & $\begin{array}{l}\text { Marginal change in } \\
\text { fluorescence }\end{array}$ \\
\hline 37 & Negative sample & $\begin{array}{l}\text { Marginal change in } \\
\text { fluorescence }\end{array}$ \\
\hline 38 & Negative sample & $\begin{array}{l}\text { Marginal change in } \\
\text { fluorescence }\end{array}$ \\
\hline 39 & Negative sample & $\begin{array}{l}\text { Marginal change in } \\
\text { fluorescence }\end{array}$ \\
\hline 40 & Negative sample & $\begin{array}{l}\text { Marginal change in } \\
\text { fluorescence }\end{array}$ \\
\hline
\end{tabular}




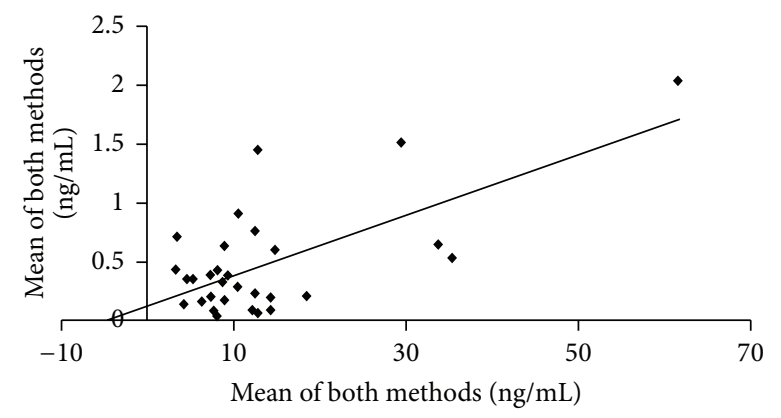

(a)

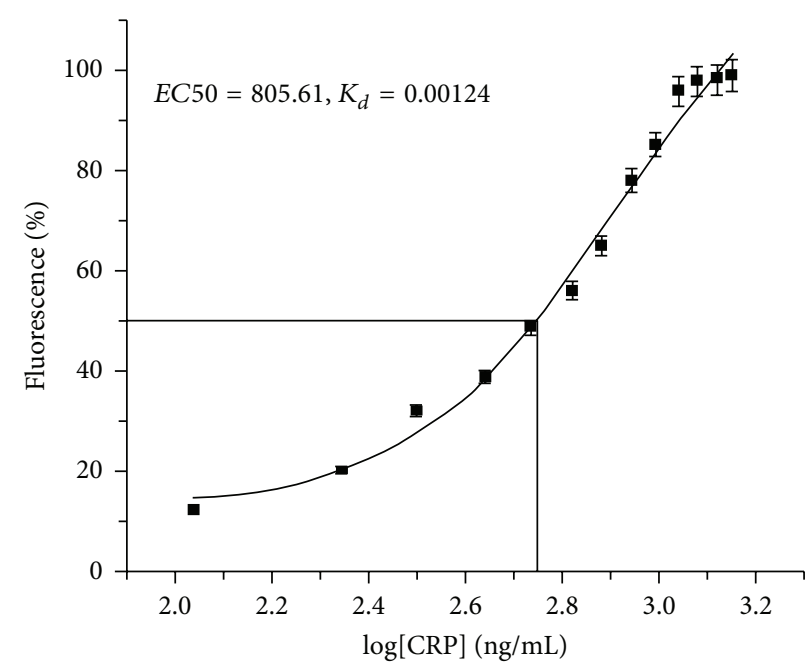

(b)

Figure 7: (a) Bland and Altman plots for measurement performed with nephelometry and fluorescence on human blood sera and (b) doseresponse curve.

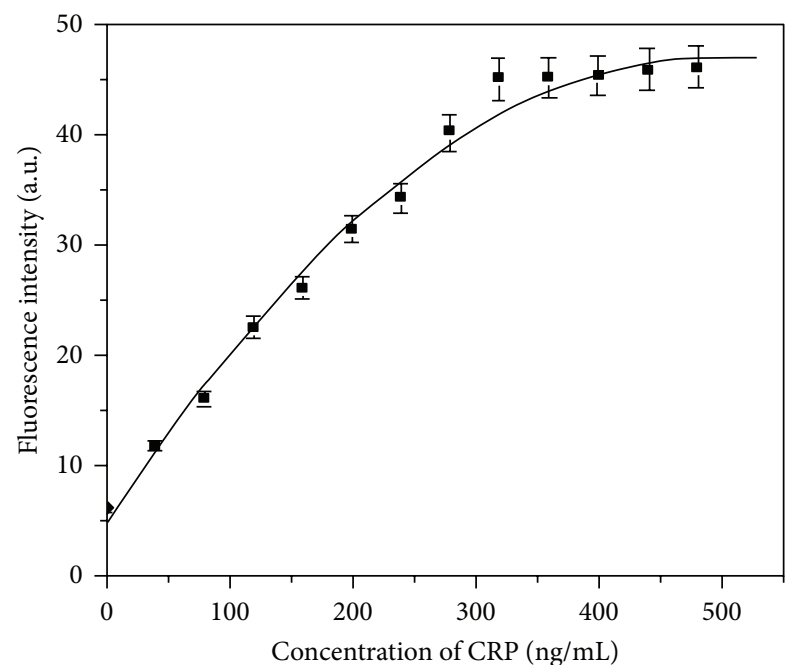

FIGURE 8: Graphical representation of variation of fluorescence intensity with concentration of CRP.

$K_{d}$ was found to be $1.24 \times 10^{-3} \mathrm{ng} / \mathrm{mL}$. The low value of affinity constant indicates good binding affinity of PEA towards CRP.

3.8. Quantification of CRP. The fluorescence measurements of polyelectrolyte at different CRP concentrations were monitored. It was seen that, with increase in CRP concentration, the fluorescence intensity increases up to a certain level and ultimately it levels off indicating the saturation of the available binding sites for CRP (Figure 8). A linear relationship between concentration of CRP and fluorescence intensity was observed until the entire CRP was bound to PEA. Further addition of protein did not show any variation in fluorescence. A linear relationship was established in the range of 40-360 ng/mL. Further addition of CRP did not cause any change in fluorescence. So the present methodology could be useful for the detection of CRP in the range of $40-360 \mathrm{ng} / \mathrm{mL}$ with a detection limit of $30 \pm 2 \mathrm{ng} / \mathrm{mL}$. The lowest amount of CRP that can produce a variation in the fluorescence intensity was $30 \pm 2 \mathrm{ng} / \mathrm{mL}$ which was taken as the detection limit of the method.

\section{Conclusion}

A new bioanalytical platform integrating the properties of polyelectrolyte and fluorescence quenching mechanism for the detection of C-reactive protein has been presented. Polyelectrolyte containing specific ligand for CRP and fluorescamine were synthesised. The fluorescence emission of fluorophore conjugated polyelectrolyte was found to decrease in presence of polyelectrolyte containing CRP specific ligand. The substantial reduction in the fluorescence intensity was attributed due to the strong polymer-polymer interactions. The fluorescence intensity was, however, found to increase in presence of CRP indicating the possibility of using this system for the detection and estimation of CRP. Other blood proteins were found not to have any interference in the measurement. The method exhibited a linear relationship in the range of $40-360 \mathrm{ng} / \mathrm{mL}$ with a detection limit of $30 \pm$ $2 \mathrm{ng} / \mathrm{mL}$. Fluorescence measurements were used to estimate CRP in serum and the results showed good correlation with hospital measurements by nephelometry. Although this work demonstrated the detection of a clinically interesting plasma protein in serum, the sensor platform described here could be modified for the detection of other clinically interesting substances. In addition to the model studied, the ease of performance, very less instrumentation, and low cost suggest broad applicability of this method. Future studies could be focussed to integrate the findings to the level of a technology, so that it could be adopted into any clinical laboratory where resources are minimal to build high tech infrastructure. 


\section{Conflict of Interests}

The authors declare that there is no conflict of interests regarding the publication of this paper.

\section{Acknowledgment}

Vidya Raj is grateful to the Council of Scientific and Industrial research (CSIR), India, for the Senior Research Fellowship.

\section{References}

[1] M. Schlupp, T. Weil, A. J. Berresheim, U. M. Wiesler, J. Bargon, and K. Muller, "Polyphenylene dendrimers as sensitive and selective sensor layers," Angewandte Chemie International Edition, vol. 40, pp. 4011-4015, 2001.

[2] D. Tyler McQuade, A. E. Pullen, and T. M. Swager, "Conjugated polymer-based chemical sensors," Chemical Reviews, vol. 100, no. 7, pp. 2537-2574, 2000.

[3] L. A. Samuelson, D. L. Kaplan, J. O. Lim, M. Kamath, K. A. Marx, and S. K. Tripathy, "Molecular recognition between a biotinylated polythiophene copolymer and phycoerythrin utilizing the biotin-streptavidin interaction," Thin Solid Films, vol. 242, no. 1-2, pp. 50-55, 1994.

[4] K. Faïd and M. Leclerc, "Functionalized regioregular polythiophenes: towards the development of biochromic sensors," Chemical Communications, no. 24, pp. 2761-2762, 1996.

[5] M. Hiller, C. Kranz, J. Huber, P. Bauerle, and W. Schuhmann, "Amperometric biosensors produced by immobilization of redox enzymes at polythiophene-modified electrode surfaces," Advanced Materials, vol. 8, pp. 219-222, 1996.

[6] G. Sukhorukov, A. Fery, and H. Möhwald, "Intelligent microand nanocapsules," Progress in Polymer Science, vol. 30, no. 8-9, pp. 885-897, 2005.

[7] C. S. Peyratout and L. Daehne, "Tailor-made polyelectrolyte microcapsules: from multilayers to smart containers," Angewandte Chemie International Edition, vol. 43, pp. 3672-3678, 2004.

[8] C. K. Ober and G. Wegner, "Polyelectrolyte-surfactant complexes in the solid state: facile building blocks for self-organizing materials," Advanced Materials, vol. 9, no. 1, pp. 17-31, 1997.

[9] P. T. Hammond, "Form and function in multilayer assembly: new applications at the nanoscale," Advanced Materials, vol. 16, no. 15, pp. 1271-1293, 2004.

[10] Q. Zhou and T. M. Swager, "Fluorescent chemosensors based on energy migration in conjugated polymers: the molecular wire approach to increased sensitivity," Journal of the American Chemical Society, vol. 117, no. 50, pp. 12593-12602, 1995.

[11] J. E. Volanaskis, "Human C-reactive protein: expression, structure, and function," Molecular Immunology, vol. 38, pp. 189-197, 2001.

[12] I. Kushner, M. L. Broder, and D. Karp, "Control of the acute phase response. Serum C-reactive protein kinetics after acute myocardial infarction," Journal of Clinical Investigation, vol. 61, no. 2, pp. 235-242, 1978.

[13] Q. Zen, W. Zhong, and R. F. Mortensen, "Binding site on human C-reactive Protein (CRP) recognized by the Leukocyte CRPreceptor," Journal of Cellular Biochemistry, vol. 64, pp. 140-151, 1997.
[14] M. O. Pentikäinen, K. Öörni, M. Ala-Korpela, and P. T. Kovanen, "Modified LDL-trigger of atherosclerosis and inflammation in the arterial intima," Journal of Internal Medicine, vol. 247, no. 3, pp. 359-370, 2000.

[15] T. B. Ledue, S. E. Poulin, L. F. Leavitt, and A. M. Johnson, "Evaluation of a particle-enhanced immunoassay for quantifying Creactive protein," Clinical Chemistry, vol. 35, no. 9, pp. 20012002, 1989.

[16] S. Birnbaum, C. Uden, C. G. M. Magnusson, and S. Nilsson, "Latex-based thin-layer immunoaffinity chromatography for quantitation of protein analytes," Analytical Biochemistry, vol. 206, no. 1, pp. 168-171, 1992.

[17] S. Nilsson, C. Lager, T. Laurell, and S. Birnbaum, “Thin-layer immunoaffinity chromatography with bar code quantitation of C-reactive protein," Analytical Chemistry, vol. 67, no. 17, pp. 3051-3056, 1995.

[18] X. Chu, J. Jiang, G. Shen, and R. Yu, "Simultaneous immunoassay using piezoelectric immunosensor array and robust method," Analytica Chimica Acta, vol. 336, no. 1-3, pp. 185-193, 1996.

[19] R. E. Banks, "Measurement of cytokines in clinical samples using immunoassays: problems and pitfalls," Critical Reviews in Clinical Laboratory Sciences, vol. 37, no. 2, pp. 131-182, 2000.

[20] J. L. Bock, "The new era of automated immunoassay," American Journal of Clinical Pathology, vol. 113, no. 5, pp. 628-646, 2000.

[21] P. M. Ridker, M. Cushman, M. J. Stampfer, R. P. Tracy, and C. H. Hennekens, "Plasma concentration of $\mathrm{C}$-reactive protein and risk of developing peripheral vascular disease," Circulation, vol. 97, no. 5, pp. 425-428, 1998.

[22] C. M. Merritt and J. W. Winkelman, "Electrochemical method for measuring C-reactive protein using crown ether-phosphate ester ionophores," Analytical Chemistry, vol. 61, no. 21, pp. 2362$2365,1989$.

[23] M. Sivakumar and K. Panduranga Rao, "In vitro release of ibuprofen and gentamicin from PMMA functional microspheres," Journal of Biomaterials Science, Polymer Edition, vol. 13, no. 2, pp. 111-126, 2002.

[24] Y. Takeoka, K. Sasada, Y. Nishiwaki, M. Rikukawa, and K. Sanui, "Fabrication of polycondensed multilayer thin films by a selfassembly method," Colloids and Surfaces A, vol. 257-258, pp. 485-488, 2005.

[25] J. B. Lee, D.-J. Kim, J.-W. Choi, and K.-K. Koo, "Formation of a protein monomolecular layer by a combined technique of $\mathrm{LB}$ and SA methods," Colloids and Surfaces B, vol. 41, no. 2-3, pp. 163-168, 2005.

[26] V. Kabanov, A. Zezin, V. Izumrodov, T. Bronich, and K. Bakeev, "Cooperative interpolyelectrolyte reactions," Die Makromolekulare Chemie, vol. 13, pp. 137-155, 1985.

[27] S. J. Dwight, B. S. Gaylord, J. W. Hong, and G. C. Bazan, "Perturbation of fluorescence by nonspecific interactions between anionic poly(phenylenevinylene)s and proteins: implications for biosensors," Journal of the American Chemical Society, vol. 126, no. 51, pp. 16850-16859, 2004.

[28] S. Shi and F. Wudl, "Synthesis and characterization of a watersoluble poly(p-phenylenevinylene) derivative," Macromolecules, vol. 23, no. 8, pp. 2119-2124, 1990.

[29] J. Park, S. Kurosawa, J. Watanabe, and K. Ishihara, "Evaluation of 2-methacryloyloxyethyl phosphorylcholine polymeric nanoparticle for immunoassay of C-reactive protein detection," Analytical Chemistry, vol. 76, no. 9, pp. 2649-2655, 2004. 

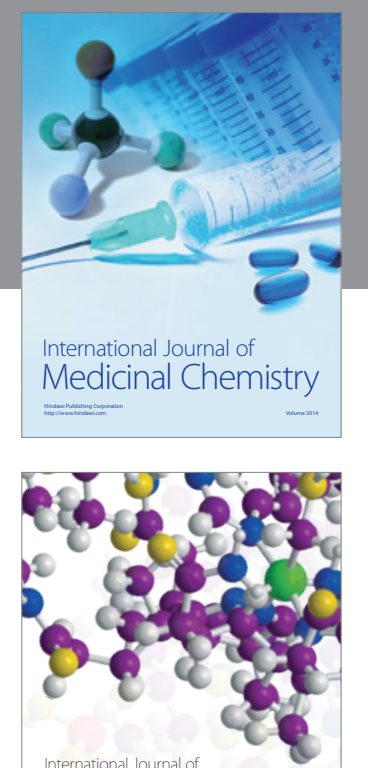

\section{Carbohydrate} Chemistry

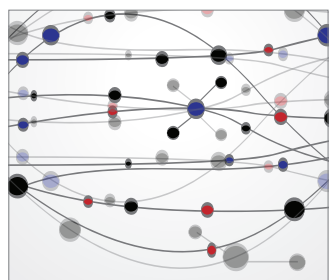

The Scientific World Journal
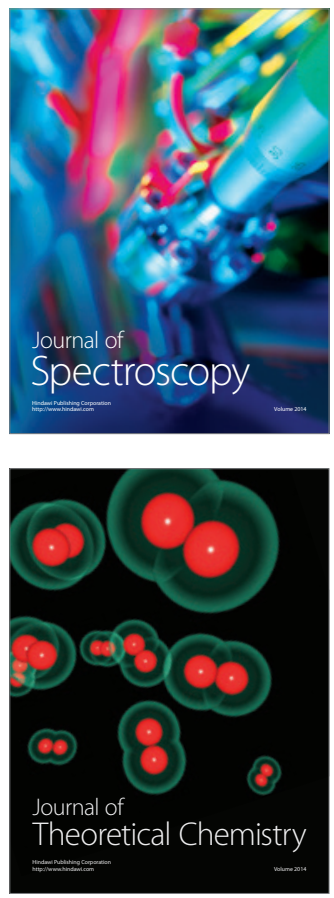
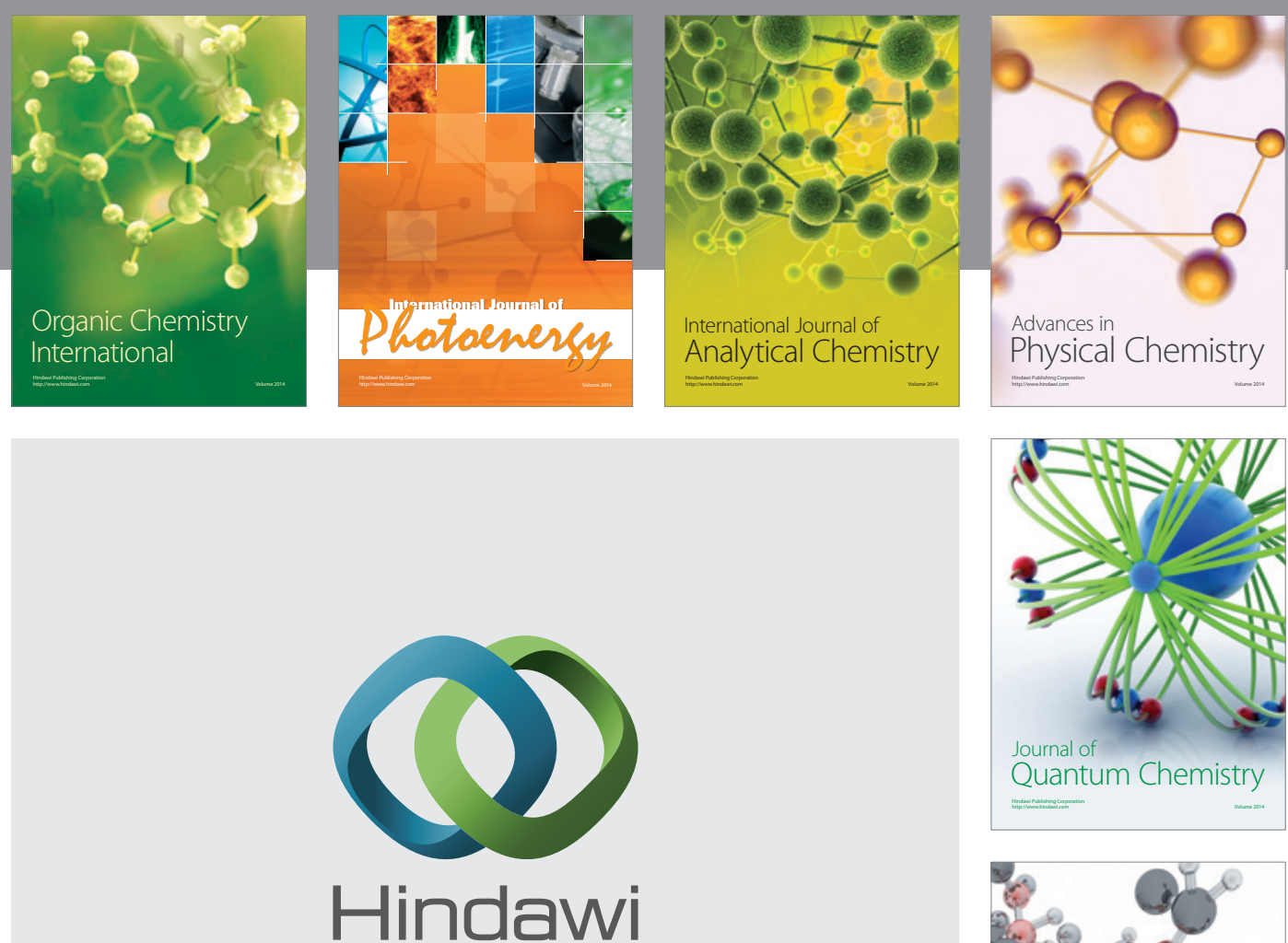

Submit your manuscripts at

http://www.hindawi.com

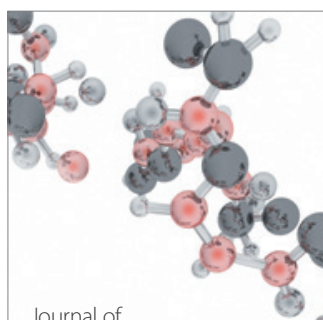

Analytical Methods

in Chemistry

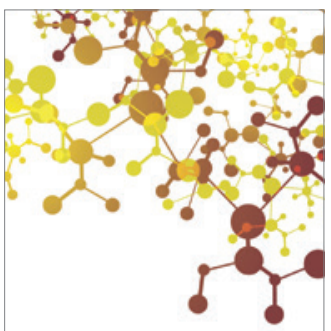

Journal of

Applied Chemistry

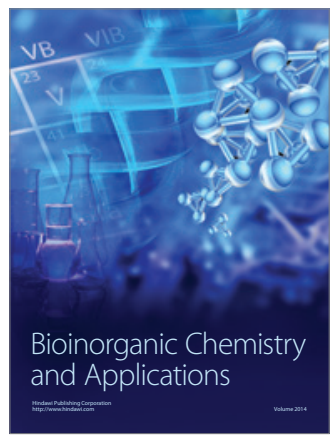

Inorganic Chemistry
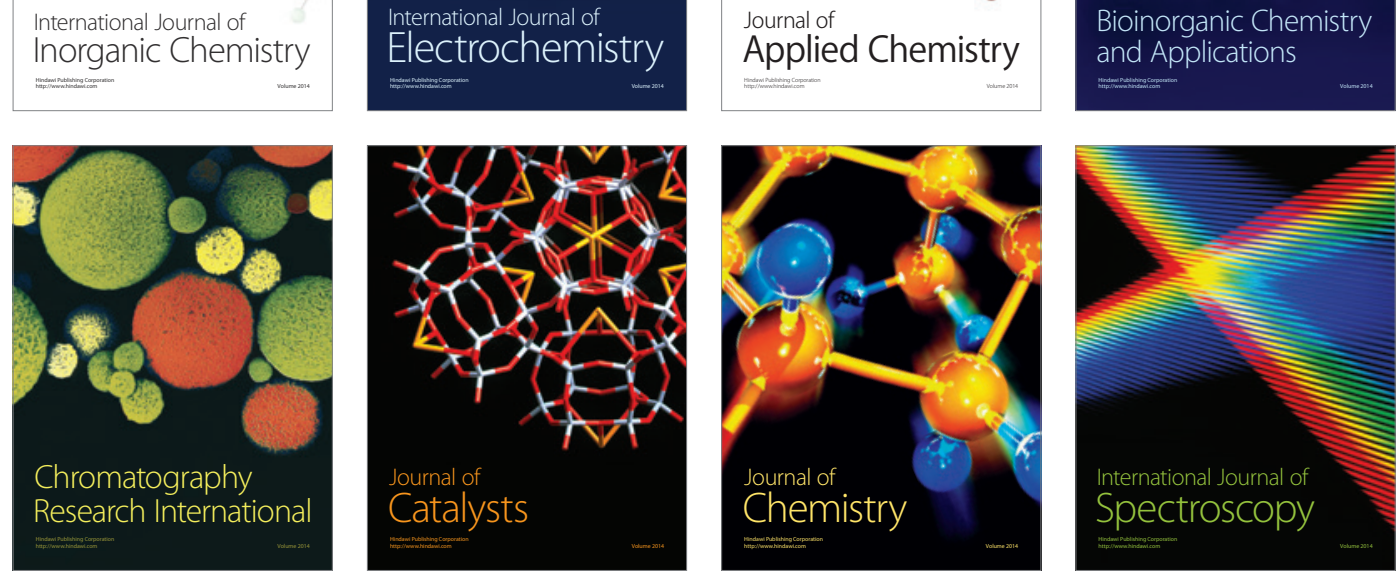